\title{
Image Mosaics Technology for Video Sequence of Inspecting Security of Pit Shaft
}

\author{
Cai Zhaohui ${ }^{\text {a, }}$, Wu Qingfeng ${ }^{\text {a }}$, Liu Shubo ${ }^{\text {a }}$ \\ ${ }^{a}$ School of Computer, Wuhan University, Wuhan, China
}

\begin{abstract}
In the vision technique and the wide visual seeing surveillance system; it always happened to actualize multiple images, non-slot merging. Phase Correlation Algorithm (PCA) is widely used as image matching method. Based on lots of simulation of phase correlation algorithm, it makes the image mosaics improve in the computing speed and accuracy.
\end{abstract}

Index Terms: Phase correlation; Inspecting of pit shaft; video sequence; Image registration

(C) 2011 Published by MECS Publisher. Selection and/or peer review under responsibility of the Research Association of Modern Education and Computer Science.

\section{Introduction}

Since 1987, the East China mining area, such as Xuzhou, Datun, Huaibei, Yanzhou, and Yongxia so on, has had a number of security incidents caused by the rupture of the shaft wall. These seriously affect the safety about normal production of minerals, causing enormous economic losses. Thus the security monitoring of the shaft is more and more important in the safe production of mine. The shaft monitoring has many ways, however, in which the video monitor is the most intuitive and easy way to achieve. In video monitor system, in order to facilitate storage management and conduct of post-image recognition, we must make mosaics for video sequence images [10].

The image mosaic is a technology, which gets the number of adjacent digital images together into an image. We must make geometric correction and registration for images to be spliced, and then we can make image mosaic on this basis. It is image registration that making images coming from different times, different perspectives or different sensors aligned in the same scene images [6]. It mainly uses for remote sensing image processing, medical image analysis, computer vision and other fields. Image mosaic is a process which gets many images together, based on the image registration. The process of image mosaic usually is made up of three steps by splicing process, image registration and stitching. The process of image mosaics is illustrated in Fig.1 (for example with two images to be spliced).

\footnotetext{
* Corresponding author.
}

E-mail address: zhcai@whu.edu.cn 


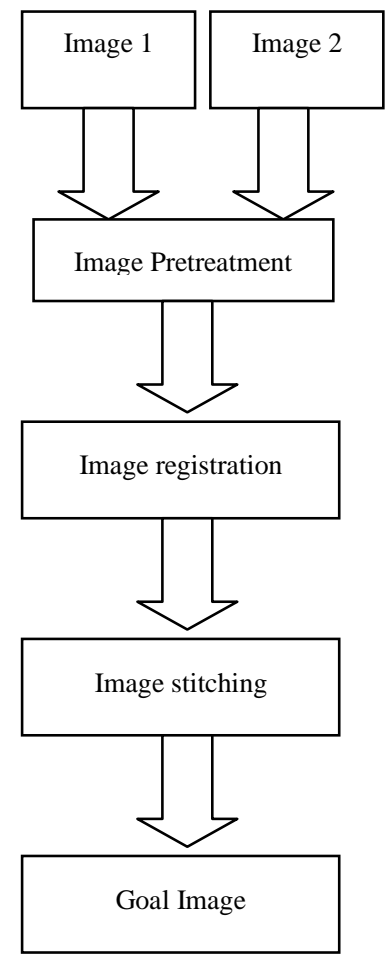

Figure 1: the process of image mosaics

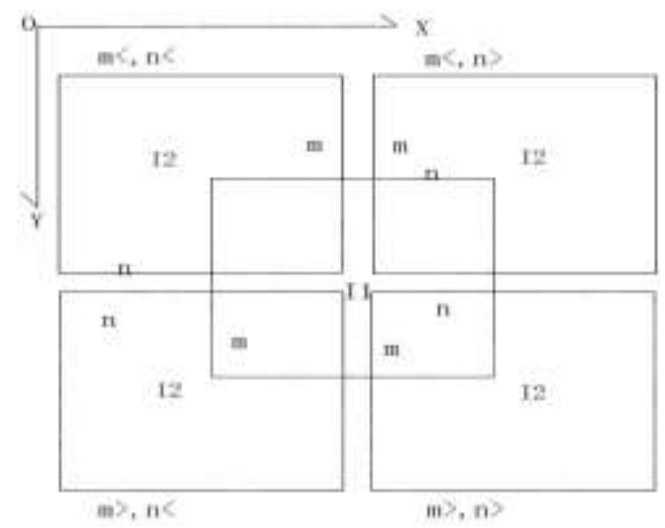

Figure 2: Two Image Position Relations

The main purpose of the splicing pretreatment is a preparative for the next step image registration, and makes the picture quality be able to meet the request of the image registration. The image registration mainly does draw the matching information between the images in which the one is used to be referred and the other is used to be splicing, then finds the best match in these information, and completes alignment between the images. The image stitching makes the superposition region of the images to be splicing together, and get a splicing restructuring smooth seamless panoramic picture [8]. 


\section{Phase Correlation Method}

Whether the image splicing is successful is depend on whether the image mosaic is accurate. The images to be spliced may have the translation, the revolving, the zoom and many other kinds of transformations or the problem that the big area's homochromy region is very difficult to match; a good image registration algorithm should be able to accurately find the corresponding information and aligned the images between the images in each kind of situation. The image registration is a key technology of the image mosaics.

Image registration has two different methods. The one is based on image gray level and the other is based on image feature. At present, the main matching method includes the control point method, the curve method, the surface method, the square and the main axle method, the correlation method (time domain, frequency rage), the most mutual information method and the nonlinear transformation technique and so on [7]. The feature extraction method involve generally a large number of geometric calculation and image morphology calculation, because of a large accounting, we must choose respective suitable feature according to different applications. But the method based on gray value is very simple, and its mathematical statistical model, convergence speed, positioning accuracy, and error estimation have quantitative analysis and research result, such methods have been generally applied. At present, the image registration algorithm which is studied most extensively is the phase correlation methods.

The phase correlation methods were earliest proposed by Kuglin and Hines in 1975.it has the independency of the scene, and can align accurately the pure two-dimensional translation image. Afterward, De Castro and Morandi discovered to determine the revolving alignment as same as the translation alignment with Fourier translation; in 1996, Reddy and Chaterji improved the De Castro algorithm, and reduced greatly quantity which needs to transform. Two images' translation vectors may directly figure out through their cross power spectrum phase [5]. Carrying on the image registration with Fourier transformation [1] is the research result of the image mosaic domain, and along with fast Fourier algorithm's statement and the mature application of the signal processing domain to Fourier transformation, the image splicing technology also obtained the corresponding development.

Phase related method [4] only solves question about the image registration of translation parameter at first. We suppose that $f_{1}(x, y)$ and $f_{2}(x, y)$ are two image signals, they meet the relation of (1) (i.e. $f_{2}(x, y)$ undergoes the simple translation by $f_{1}(x, y)$ to obtain).

$$
f_{2}(x, y)=f_{1}\left(x-x_{0}, y-y_{0}\right)
$$

We carry separately on the Fourier transformation to $f_{1}(x, y)$ and $f_{2}(x, y)$, and can get result by Fourier transformation's nature:

$$
F_{2}(u, v)=F_{1}(u, v) e^{-j\left(u x_{0}+v y_{0}\right)}
$$

$F_{1}(u, v)$ and $F_{2}(u, v)$ in (2) respectively are the Fourier transformation of $f_{1}(x, y)$ and $f_{2}(x, y)$. Their cross-power spectrum is

$$
\frac{F_{1}^{*}(u, v) F_{2}(u, v)}{\left|F_{1}^{*}(u, v) F_{2}(u, v)\right|}=e^{-j\left(u x_{0}+v y_{0}\right)}
$$

In the formula (3), $F_{1}^{*}(u, v)$ is the duplicate conjugate of the $F_{1}(u, v)$; the Fourier inverse transformation of the $e^{-j\left(u x_{0}+v y_{0}\right)}$ is a two-dimensional impulsive function $\delta\left(x-x_{0}, y-y_{0}\right)$. The phase correlation method is that we seek the Fourier inverse transformation of (3), and find the peak value position of the impact function, then determined the translation parameter $x_{0}$ and $y_{0}$. 


\section{Image Mosaic in Video Sequence}

The primitive phase correlation method has the very high precision, when it processes the translation of only two images. The error is between 1-2 picture elements. But it has two problems in video sequence's mosaic, one is the determination of the position relations, the other is the boundary fissure of the image mosaic.

\section{A. Position relations determination}

Because there is the large redundant information between the frames of the video stream, therefore we must carry on the concentration to the video data, and one method in these can make the separate information in each video frame concentrate to show the whole scene through the image mosaics technology, then may realize some effective direct operations to the video data such as searching, browsing, edition and so on. Because the splicing images have represented the public information of all the frames, we can explain each frame separately in turn, this kind of close reorganization provides the misalignment and the highly effective index to the content, and can effectively do the direct quick access to the information interested by us. On making the video retrieve, above all we search the splicing image, and find the goal which we need, then use the geometry localization mechanism, in the end; we can very conveniently retrieve the corresponding video frame.

We suppose the splicing image is $I_{1}$ and $\boldsymbol{I}_{2}$, using phase correlation method can only simple extract the relative displacements of two images, and cannot specifically define the relative positions of two images. After two images extracting relatively horizontal and vertical displacement $\mathrm{m}$ and $\mathrm{n}$, the possible of position relations of two images are: Like Fig.2. That $I_{2}$ is opposite in $I_{1}$ has 4 possible positions. Only according to the horizontal and vertical displacement, we cannot define which relative position $I_{2}$ is in $I_{1}$.

Video data each second is composed of 25-30 frames. After analyzing and counting the decomposition video sequence, we find the overlap between the sequence images is least big than $25 \%$, namely the overlap region's length and breadth will be bigger than the $1 / 2$ of the image's length and breadth. We may compare $\mathrm{m}$ and $n$ value of the relative positions and the $1 / 2$ of the picture's length and breadth, thus determines the relative position of the overlap region.

\section{B. Boundary Fusions}

After obtaining the space relations of two images, in order to obtain the composite image, we need to choose the appropriate boundary stitching strategy, and completes the image mosaics. The image mosaic is not the simple image superimposition, but generates the new image which has the many most value information. The image mosaics is one kind technology that it mixes the multiple source images through the high-level image processing, and uses specific algorithm to make two or more different images merge, in the end, we can get the new image. Its goal is which reduces the uncertainty of the pictorial information as far as possible, namely it synthesizes the information provided by the different images, eliminates the redundancy and contradiction which possibly exist between the pictorial information, and gets the clear, complete and accurate information description to the goal.

Toward the overlap region of the image, if we only carry on simply the average weighting summation, it will exist obviously the mark belt in the splicing image, the primary cause is the difference of brightness between the neighboring image and the ordinary camera's photography in the images which were gathered will exist the phenomenon of the edge distortion, but which area the mosaic uses is the fringe area of image [2]. The article uses the function method of weighting to process the overlap region of image, and guarantees the seamless transition of image.

Suppose $I_{1}(x, y)$ and $I_{2}(x, y)$ respectively are the picture element value of image region $((x, y)$ spot $)$ to be fused, then after fusing, this spot picture element value $I(x, y)$ is: 


$$
I(x, y)=W_{1}(x, y) I_{1}+W_{2}(x, y) I_{2}
$$

And $\mathrm{x}$, y respectively is the overlap picture element point corresponding to the weighted value of I1 and I2. In order to cause the image can transit smoothly in2direction after splicing the image, and achieve the better fusion effect [9], the article's weighting factors W1 $(x, y), W 2(x, y)$ use the function of the gradual enter and leave.

$$
\begin{aligned}
& W_{1}(x, y)=i / a \\
& W_{2}(x, y)=1-W_{1}
\end{aligned}
$$

And ' $a$ ' is the height of the superposing region, ' $i$ ' is the along distance from current spot to superposition region.

\section{Result and Analysis}

The article uses the Tri-Star camera matching four angles bracket to carry on the fixed photography to the iron ore well chamber facility, the camera along with the hanging basket low went downward by a low speed, and recorded 2 minutes. Then we selected the quite good photography effect as the sample, and made the video sequence decompose 14 sequence images, we use the algorithm of this article to carry on the matching splice for the sequence image [3], Like Fig.3. And Fig.3 is the sample sequence image. Fig.4 is the splicing effect chart which uses the algorithm of this article.

Before the splicing, we carry on pretreatment work to the photography sequence image, such as noise elimination, adjustment and so on; these improve the image frequency range relevance effectively, and increased the image matching precision. In image overlap area, we used smooth splicing, carried on processing the image brightness to eliminate the boundary trace, and improved the splicing quality.

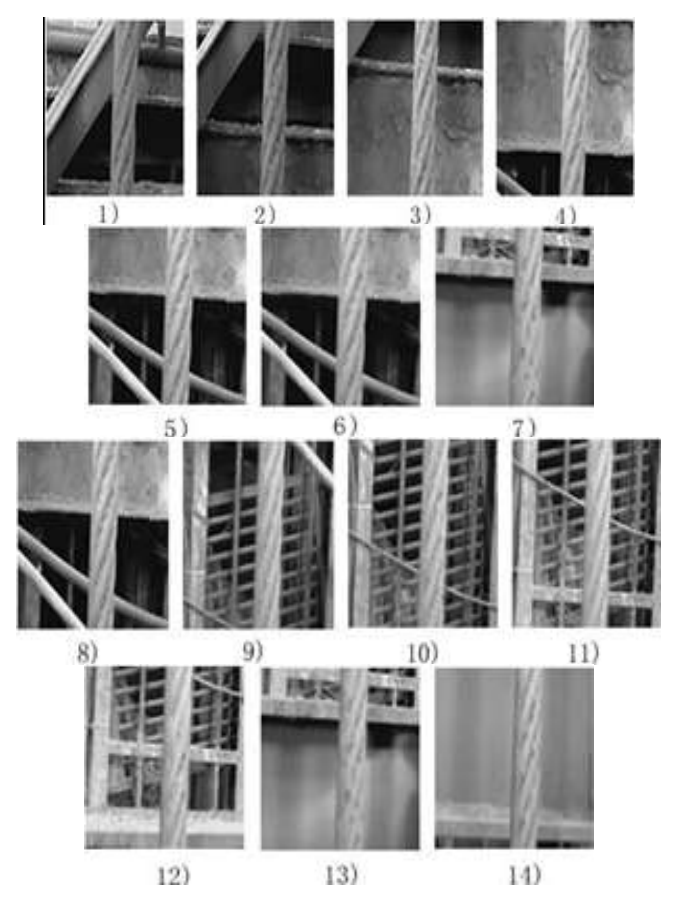

Figure 3: 14 papers of video sequence chart 


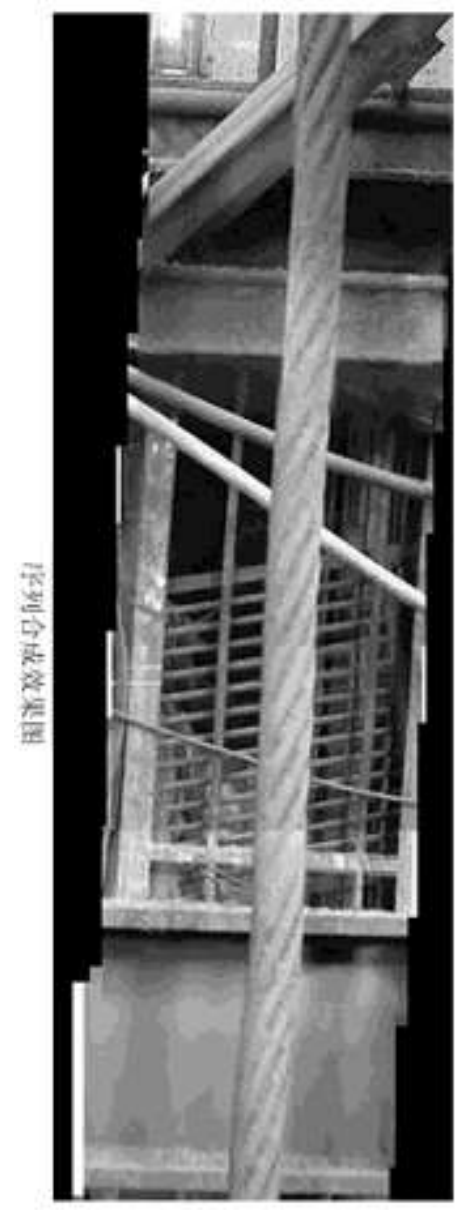

Figure 4: Synthesis effective chart

This article states the application of the splicing method in the well chamber observation system. On the one hand it effectively reduces the size of the monitor video material, conveniences the management of system, and has simultaneously convenient for the browsing of the user. On the other hand it provides the foundation for extracting the wall depth mark in the well chamber image in the later period.

\section{References}

[1] DeCastro, E., Morandi, C., 1987. Registration of translated and rotated images using finite Fourier transforms. IEEE Transactions on Pattern Analysis and Machine Intelligence95, pp.700-703

[2] Gibson, S., 1999. An optimal FFT-based algorithm for mosaic image. M.S. Thesis, University of Texas at EI Pasp,Tx, pp.171.

[3] Aitmann, J., Reitbock, H.J.P.: A Fast Correlation Method for Scale- and Translation-Invariant Pattern Recognition. IEEE Trans. on Pattern Analysis and Machine Intelligence, Vol. 6. (1984) pp. 46-57.

[4] Casasent, D., Psaltis, D.: Deformation Invariant, Space-Variant Optical Pattern Recognition. In: Wolf, E. (ed.): Progress in Optics, North-Holland Publishing Co, Amsterdam (1978) pp.290-356. 
[5] DeCastro, E., Morandi, C.: Registration of Translated and Rotated Images Using Finite Fourier Transforms. IEEE Trans. on Pattern Analysis and Machine Intelligence, Vol. 9, No. 5. (1987) pp.700-703

[6] QiangZanxia, PengJiaxiong, WangHongqun. Image registration algorithm based on the remote sensing Fourier transform [M], On Infrared and Laser Engineering (2004), pp.385-387, (in Chinese).

[7] ShuXianbiao, NiGuoqiang, ZhengWei, LiJiangtao. Image registration algorithm based on detection of the phase correlation [M], On Optical Technology (2007), pp.859-862,(in Chinese).

[8] HuShejiao, GeXiwan, SuLing. Panoramic image mosaics based on the phase correlation [M], On Hefei University of Technology Journal (2007), pp.29-32, (in Chinese)

[9] YangGang. The Design and Implementation of Panoramic Image Stitching Algorithm [M]. On Chongqing Institute of Technology Journal (2007), pp.107-110, (in Chinese).

[10] QiChi, LiuQing. The panorama stitching image sequence of the camera [M], On Computer Assist Design and Computer Graphics (2001), pp.605-609, (in Chinese) 